\title{
L'empreinte génomique parentale
}

Dans son acception la plus large, le terme d'empreinte génomique se rapporte au fait qu'un phénotype contrôlé par un gène ou un groupe de gènes se manifeste en fonction de l'origine parentale du gène ou $d u$ groupe de gènes en question. Ce terme a été utilisé pour la première fois par Crouse en 1960 [1], pour rendre compte du mode très particulier de détermination du sexe d'un invertébré, l'insecte Sciara : en effet chez cet animal, le zygote contient trois chromosomes X (deux paternels et un maternel) et, après quelques divisions du zygote, soit un, soit deux chromosomes paternels sont spécifiquement éliminés, ce qui donne naissance, soit à une femelle, soit à un mâle. Crouse concluait à l'existence d'une "empreinte génomique " qui permettrait, d'une manière ou d'une autre, à la cellule de " reconnaître " l'origine parentale des chromosomes à éliminer. Le cas de Sciara n'est pas limitatif : des phénotypes variés dans différentes espèces peuvent dépendre d'une empreinte génomique ; cependant, nous nous en tiendrons dans ce qui va suivre au cas des mammiferes chez lesquels, comme nous le verrons, l'empreinte génomique prend une signification particulière, car elle conditionne la survie de l'embryon. Il faut tout d'abord noter que si la reproduction sexuée (à partir d'un zygote, fruit de la rencontre d'un gamète mâle et d'un gamète femelle) est la règle chez les eucaryotes supérieurs, des cas de reproduction asexuée (à partir de gamètes d'un seul des deux parents) sont documentés chez des espèces appartenant à tous les groupes taxonomiques, sauf celui des mammifères. Chez la souris, toutes les tentatives visant à obtenir le développement complet à partir d'œufs parthénogénotes* (PG) ou gynogénotes* contenant uniquement un génome femelle se sont soldées $\mathrm{m} / \mathrm{s} n^{\circ} 1$, vol. 8 , janvier 92 par un échec. Bien qu'un résultat négatif soit toujours d'interprétation délicate, ces expériences suggéraient l'incapacité d'un seul génome d'origine maternelle à assurer le développement complet d'un embryon. Cependant, qu'en était-il du seul génome paternel ? Pour répondre à cette question, il a fallu attendre le développement par McGrath et Solter [2] d'une méthode permettant de transférer, à volonté, d'un zygote* à l'autre, un pronoyau* (PN) d'origine paternelle ou maternelle. Des zygotes uniparentaux maternels (gynogénotes) ou paternels (androgénotes* $[A G]$ ont pu ainsi être créés et il s'est avéré que ni les uns ni les autres n'étaient capables de se développer à terme. En revanche, les zygotes témoins obtenus par le transfert d'un PN mâle et d'un PN femelle dans un zygote préalablement énucléé, se développaient à terme et donnaient naissance à des souris parfaitement normales et fertiles [3] (figure 1).

Ces expériences ont permis d'aboutir à plusieurs conclusions importantes : la présence simultanée d'un génome d'origine paternelle et d'un génome d'origine maternelle dans le zygote est indispensable au développement normal de l'œuf; les génomes parentaux sont donc fonctionnellement différents ; de plus, comme on sait qu'ils contiennent une information génétique globalement identique, cela amène à faire l'hypothèse qu'ils portent une empreinte différentielle qui leur a été apposée à un certain moment avant la formation des PN mâle et femelle dans le zygote (probablement lors de la formation de la lignée germinale), empreinte qui rendrait compte de leur différence fonctionnelle [4-6]. Il faut noter que, dans

\footnotetext{
* Voir glossaire, p. 69
}

ces expériences, les génomes parentaux (paternels et maternels) sont traités comme deux entités globales qui s'avèrent fonctionnellement complémentaires. Cependant, grâce aux travaux des généticiens, et bien avant qu'on arrive aux conclusions ci-dessus mentionnées, on disposait de lignées de souris produisant des zygotes ayant un contenu chromosomique diploïde normal mais chez lesquels tout ou partie de chromosomes d'une paire d'homologues provenait d'un seul des deux parents. Or, certains de ces zygotes porteurs de duplications uniparentales particulières donnaient naissance à des embryons à phénotype anormal (pouvant aller jusqu'à la mort de l'embryon). Ces observations ont permis, non seulement de conforter l'hypothèse de la complémentarité fonctionnelle des génomes parentaux, mais encore de dresser une carte des différentes régions du génome de la souris selon qu'elles sont ou non soumises à une empreinte génomique. Environ une dizaine de régions (dont six ou sept sont localisées seulement sur trois chromosomes) ont ainsi été définies qui présentent une différence fonctionnelle selon qu'elles sont héritées du père ou de la mère [7].

On peut donc faire l'hypothèse que dans les régions génomiques en question réside(nt) un ou plusieurs gène(s) dont seul l'un des deux allèles est exprimé et ce, en fonction de l'origine parentale du chromosome qui le porte (figure 2). Le phénotype présenté par les embryons porteurs de duplications uniparentales résulterait alors de l'absence d'expression ou de l'hyperexpression de ces gènes. Il faut noter que la complémentarité fonctionnelle des génomes parentaux est probablement un phénomène qui concerne l'ensemble des mammifères: dans l'espèce humaine, par exemple, de nombreuses observations 


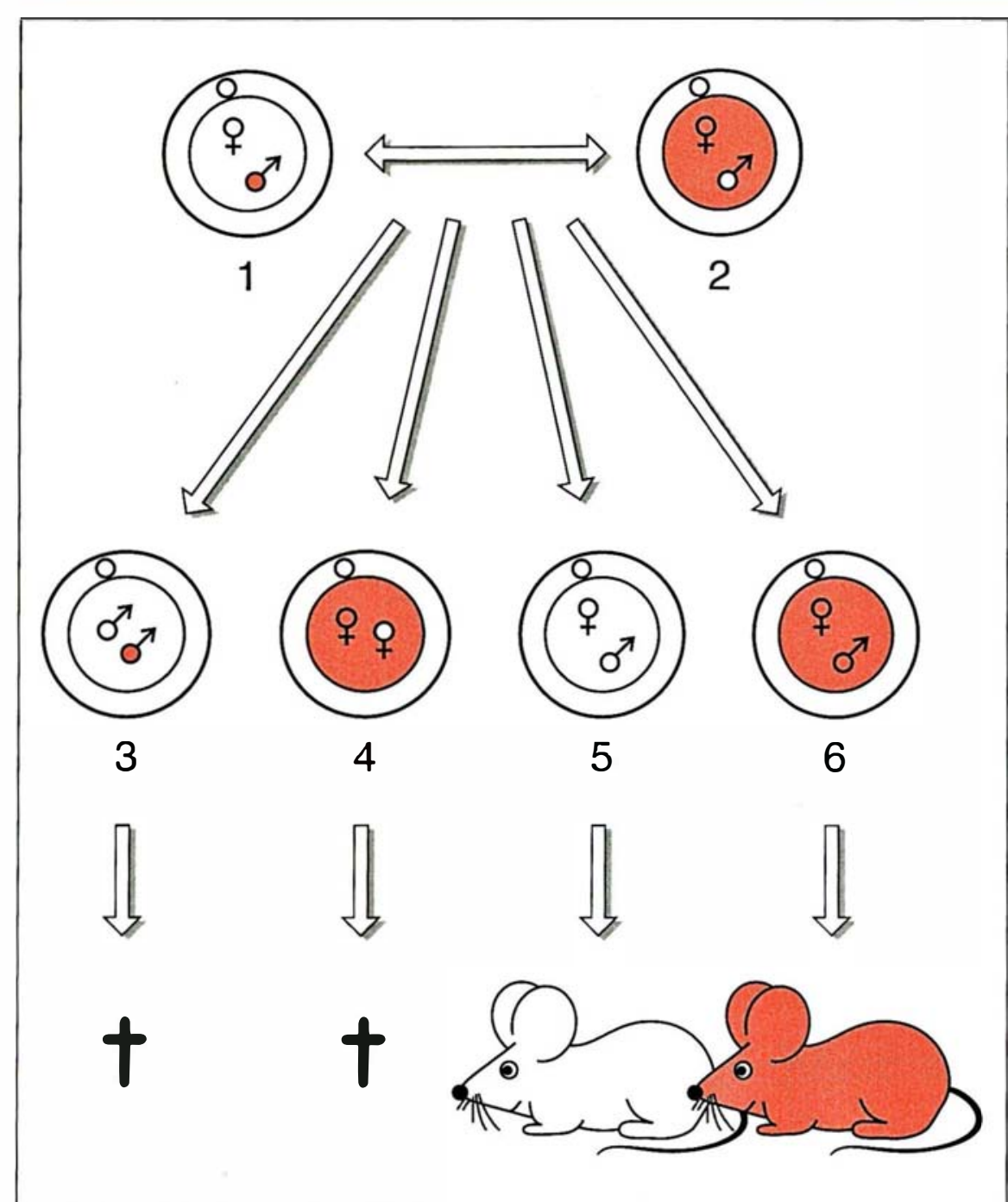

Figure 1. Démonstration de la complémentarité fonctionnelle des génomes parentaux par transfert nucléaire dans l'œuf fécondé. Les zygotes de départ sont des hybrides entre deux lignées de souris différentes, l'une représentée en rouge, l'autre en blanc. L'échange du pronoyau femelle d'un œuf fécondé [1] avec le pronoyau mâle d'un autre œuf fécondé [2] permet d'obtenir des embryons diploïdes uniparentaux mâles [3] et femelles [4]. En revanche, si l'on échange entre eux les deux pronoyaux mâles, les embryons ainsi obtenus conservent un génome biparental [5] et [6]. Seuls ces embryons donnent des nouveau-nés.

suggèrent très fortement l'existence de cette complémentarité ; ainsi le développement d'embryons contenant uniquement des chromosomes d'origine paternelle se caractérise par un conceptus constitué essentiellement de trophoblaste (moles hydatiformes) et rappelle de manière saisissante celui

(voir plus loin). D'autre part, certaines duplications uniparentales localisées, comme celles que l'on observe dans les syndromes de Prader-Willi et d'Angelman, entraînent des phénotypes particuliers ; enfin, on constate, dans certains types de tumeurs, la perte préférentielle de l'un des allèles parentaux $[8,9]$.
Le destin des cellules embryonnaires à contenu génomique uniparental : un tableau contrasté

Nous avons vu qu'en aucun cas les zygotes uniparentaux ne poursuivent leur développement à terme. Cependant, l'observation du développement limité des zygotes uniparentaux ainsi que des chimères construites par diverses combinaisons d'embryons uniparentaux et biparentaux apporte des enseignements particulièrement intéressants [10]. Les embryons uniparentaux atteignent au mieux le milieu de la gestation mais l'examen des conceptus issus de zygotes androgénotes $(\mathrm{AG})$ ou parthéogénotes $(\mathrm{PG})$ à ce stade offre un contraste frappant : alors que les conceptus AG présentent des annexes embryonnaires bien développées et un embryon extrêmement retardé, au contraire les conceptus PG contiennent un embryon apparemment normal et des annexes très peu développées. La distribution des cellules d'origine $\mathrm{AG}$ ou $\mathrm{PG}$ dans des chimères par agrégation* de type $\mathrm{AG} \leftrightarrow \mathrm{N}$ (obtenues par l'agrégation d'un embryon $A G$ avec un embryon normal) ou de type PG $\leftrightarrow \mathrm{N}$ ou $\mathrm{PG} \leftrightarrow \mathrm{AG}$ confirme cette tendance : en effet, dans les chimères des différents types examinés vers le milieu de la gestation, les cellules d'origine PG se retrouvant en grande majorité dans l'embryon, tandis que les cellules d'origine $A G$ sont cantonnées au tissu extraembryonnaire [11]. On aurait pu conclure de cette dernière observation à une incapacité des cellules $A G$ à coloniser l'embryon. Cependant, des expériences récentes remettent en cause cette conclusion $[12,13]$ : en effet la production de chimères par injection* de cellules embryonnaires androgénétiques dans un blastocyste normal (donc à un stade plus tardif que dans le cas des chimères par agrégation) a montré que celles-ci pouvaient également coloniser les tissus de l'embryon. L'ensemble des expériences sur les chimères permet de dresser un tableau assez complet des potentialités de développement des cellules AG et $\mathrm{PG}$ qui vient conforter et préciser les différences fonctionnelles entre les

Voir glossaire, p. 69. 


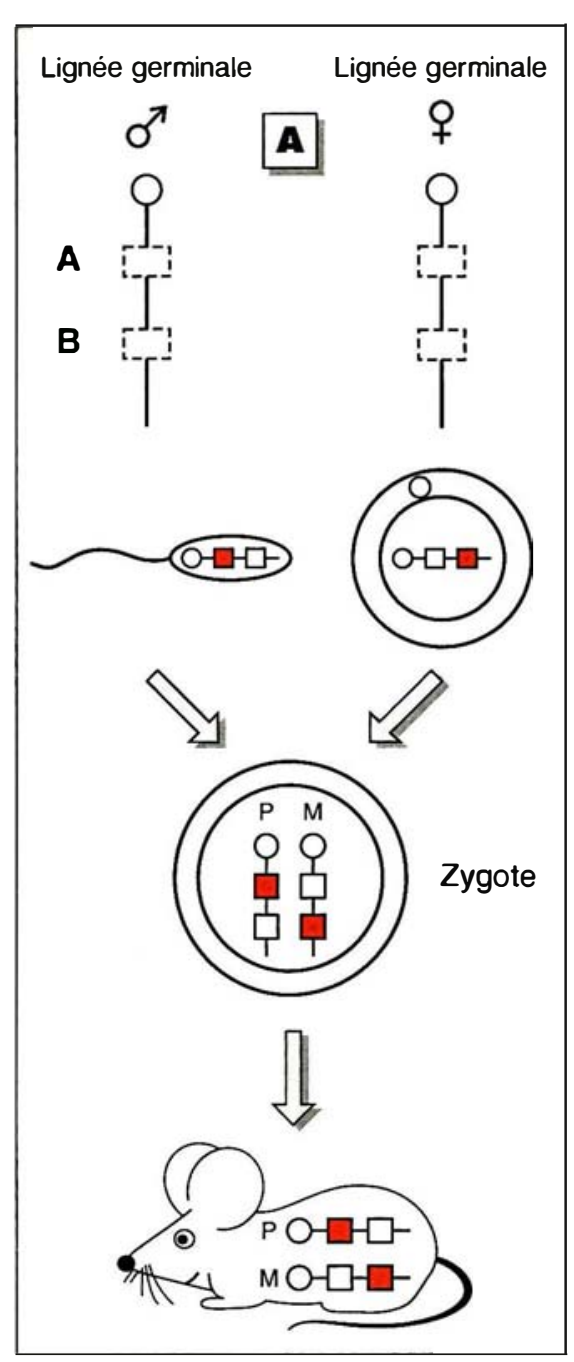

deux types de cellules uniparentales : (1) contrairement aux chimères $\mathrm{AG} \leftrightarrow \mathrm{N}$ ou $\mathrm{PG} \leftrightarrow \mathrm{N}$, les chimères $\mathrm{AG} \leftrightarrow \mathrm{PG}$ ne se développent jamais à terme, ce qui indique la nécessité de la présence simultanée des deux génomes parentaux, au moins dans un certain nombre de cellules de l'embryon [11] ; (2) les chimères $\mathrm{AG} \leftrightarrow \mathrm{N}$ sont généralement plus grandes pendant la période fotale, les chimères $\mathrm{PG} \leftrightarrow \mathrm{N}$ étant généralement plus petites que les témoins $\mathrm{N} \leftrightarrow \mathrm{N}$ [13] ; (3) les cellules PG ne colonisent pas les dérivés extraembryonnaires alors que l'inverse est observé par les cellules AG [11] ; (4) durant la vie embryonnaire, une sélection importante s'exercc contre les cellules $\mathrm{PG}$, ce qui ne semble pas être le cas pour les cellules AG [13-15] ; (5) la contribution $m / s n^{\circ} 1$, vol. 8 , janvier 92
$\mathbf{B}$

A

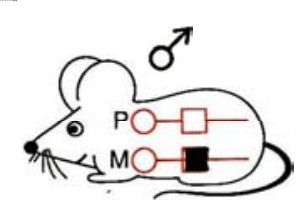

B
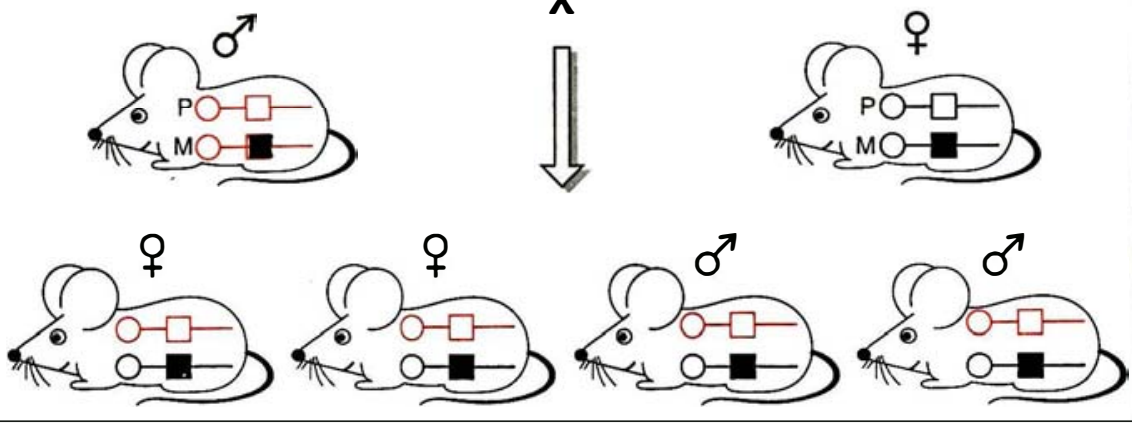

Figure 2. L'hypothèse de l'empreinte génomique parentale. (A) Pour un gène soumis à l'empreinte, l'allèle exprimé dépend de l'origine parentale du chromosome qui le porte. Cela résulte d'un marquage différentiel des chromosomes parentaux, probablement au cours de la gamétogenèse. Le marquage est stable au cours des divisions, de telle sorte que dans l'embryon ou l'adulte, l'expression spécifique du gène n'a lieu qu'à partir de l'allèle maternel (cas du gène A) ou de l'allèle paternel (cas du gène B). (B) L'hypothèse de l'empreinte génomique parentale suppose la réversibilité de celle-ci. Nous considérons le cas où seul l'allèle paternel est exprimé. Les individus issus du croisement ci-dessus ont tous un allèle maternel inactif $(\square)$ et un allèle paternel actif ( $\square$ ). II a donc fallu que les empreintes de l'allèle paternel de la femelle $B$ et de l'allèle maternel du mâle $A$ soient effacées et remplacées par une empreinte respectivement paternelle et maternelle. Ainsi tous les individus de la génération F1, qu'ils soient mâle ou femelle, portent un allèle paternel actif et un allèle maternel inactif. des cellules AG est prédominante dans les tissus dérivés du mésoderme, celle des cellules PG prédominante dans les dérivés de l'ectoderme [13-15] ; (6) enfin, il faut noter que s'il est possible d'obtenir des souris chimériques $\mathrm{PG} \leftrightarrow \mathrm{N}$ normales et fertiles (avec formation de cellules germinales issues de cellules PG), en revanche, les chimères $A G \leftrightarrow N$ présentent des anomalies graves, en particulier de l'appareil squelettique, et très peu d'entre elles survivent jusqu'à l'âge adulte. L'ensemble de ces observations souligne l'existence d'anomalies fonctionnelles différentielles des cellules possédant un génome issu d'un seul des deux parents, anomalies dues vraisemblablement à l'expression inappropriée de gènes essentiels pour la croissance et la différenciation normale de l'embryon.
Un modèle d'étude de l'empreinte génomique : la méthylation des transgènes

Le type de modification épigénétique constituant l'empreinte génomique, pas plus que le signal qui déclenche l'apposition de l'empreinte, ne sont aujourd'hui connus. Cependant, il était tentant d'examiner le statut de méthylation d'allèles parentaux lors de croisements réciproques : en effet, ce type de modification épigénétique répond aux caractéristiques attendues de l'empreinte génomique, telle que nous l'avons définie plus haut : elle peut être transmise clonalement, elle est révcrsible et, enfin, la présence ou l'absencc des sites méthylés dans un gène a pu être corrélée dans nombre de cas à son niveau d'expression. De plus, un modèle d'étude de ce type de modification épigénétique s'offrait

\section{.

.

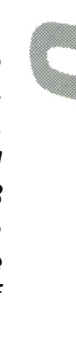

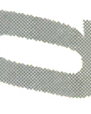

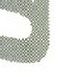

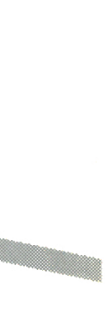


grâce à l'existence des souris transgéniques chez lesquelles le transgène, aisément repérable, peut être transmis de manière contrôlée, soit par le père, soit par la mère. De fait, dans un premier temps, l'examen de lignées transgéniques portant différents transgènes a montré que, pour environ $15 \%$ d'entre elles, le statut de méthylation du transgène était conforme à celui qu'on attend d'un gène soumis à l'empreinte génomique [16]. La méthylation était en général élevée lorsque le transgène était transmis par la mère, faible lorsqu'il était transmis par le père. De plus, un allèle paternel (faiblement méthylé) transmis par une femelle se retrouvait hautement méthylé chez sa progéniture, la situation symétrique étant observée pour un allèle maternel transmis par un mâle. Enfin, dans certains cas au moins, il y avait corrélation entre le niveau d'activité du transgène et son état de méthylation. Depuis ces premières observations, l'étude de l'empreinte par méthylation a permis de mettre à jour certains des facteurs qui gouvernent cette modification épigénétique des transgènes ainsi que leurs éventuelles conséquences sur le phénotype. En particulier, il a été montré que la méthylation de certains transgènes était contrôlée génétiquement par des gènes dits " modificateurs " et pour lesquels des allèles différents selon les lignées de souris ont pu être mis en évidence $[17,18]$. Un tel gène qui gouverne la méthylation d'un transgène donné et ce, indépendamment de sa position dans le génome, a pu ainsi tout récemment être localisé sur le chromosome 4 [19]. Fait intéressant, deux allèles de ce gène, présents dans deux lignées de souris consanguines différentes, ont été également caractérisés, l'un entraînant une méthylation élevée du transgène, l'autre une méthylation faible, ce qui démontre que des gènes "modificateurs" peuvent être polymorphes. De plus, certains de ces gènes modificateurs sont eux-mêmes soumis à l'empreinte génomique, l'activité de l'allèle modificateur dépendant du parent qui le transmet [20]. Ces deux dernières observations, si elles étaient étendues à des gènes endogènes, et pour peu que le degré de méthylation influence leur expression, pourraient servir de base à l'explication de la variation de pénétrance que l'on observe pour certaines mutations, et qui dépend à la fois du fond génétique et du sens du croisement. Enfin, phénomène particulièrement intéressant, il a été démontré que le degré de méthylation d'un transgène pouvait être différent dans différentes cellules d'un même tissu et son expression varier en conséquence [21]. De même que, dans le cas des gènes " modificateurs " décrits ci-dessus, si cette dernière observation était confirmée dans le cas des gènes endogènes, elle signifierait que dans une partie des cellules d'un individu normal, certains gènes seraient fonctionnellement hémizygotes.

L'hypothèse de l'empreinte génomique parentale suppose, nous l'avons vu, que celle-ci soit réversible lorsqu'un allèle parental est transmis au parent de sexe opposé. Différents arguments permettent de penser que cet événement a lieu au cours de la gamétogenèse. Chaillet et al. [22] ont donc suivi le diagramme de méthylation d'un transgène soumis à l'empreinte parentale, au cours de la gamétogenèse et du développement embryonnaire. Ils ont ainsi montré que le diagramme de méthylation, qu'il soit hérité du père ou de la mère, était effacé dans les cellules germinales primordiales mâles ou femelles: le transgène est alors déméthylé. C'est au cours de la maturation des cellules germinales qu'une différence s'établit : l'allèle maternel acquiert son diagramme de méthylation caractéristique (hyperméthylé) au cours de l'ovogenèse et tel qu'on le retrouvera chez l'adulte. Dans le cas de l'allèle paternel, la situation est plus complexe: il acquiert un diagramme de méthylation distinctif dans le spermatozoïde, mais ce n'est qu'après la fécondation, vers le sixième jour du développement, que les caractéristiques adultes de méthylation sont définitivement établies. Rien ne permet à l'heure actuelle de décider si les types de méthylation et leur contrôle génétique mis en évidence avec les transgènes s'appliquent aux gènes endogènes. Mais l'ensemble de ces données a permis de mettre en évidence la com- plexité des mécanismes susceptibles de moduler de manière fine la méthylation et, parfois également, l'expression de certains transgènes. La question de savoir si des mécanismes similaires sont mis en jeu dans le cas des gènes endogènes soumis à l'empreinte génomique ne devrait pas tarder à trouver sa réponse car trois d'entre eux viennent d'être mis à jour.

Des gènes de la souris soumis à l'empreinte génomique parentale A ce jour, trois gènes ont été identifiés chez la souris, dont l'expression dépend de l'origine parentale du chromosome qui les porte. Il s'agit du gène IGF2-r qui code pour l'insulin like growth factor 2 [23], du gène qui code pour le récepteur de ce même facteur de croissance (gène $I G F 2-r)[24]$ et, enfin, du gène $H 19$ [25] qui exprime un ARN messager ne semblant pas posséder de phase ouverte de lecture mais qui pourrait néanmoins avoir une fonction importante puisqu'une surexpression de ce gène dans les souris transgéniques entraîne une létalité embryonnaire [26]. La découverte de l'empreinte génomique parentale du gène $I G F 2-r$ a pour origine l'existence d'une des très rares mutations à effet maternel connues chez la souris, la mutation $T^{\text {hp }}$ (pour $T$ hairpin, car les hétérozygotes pour cette mutation ont une queue raccourcie et tordue, d'une forme rappelant l'épingle à cheveux !). Cette mutation, une délétion, est létale lorsqu'elle est transmise par la mère, en revanche, elle n'induit pas de phénotype lorsqu'elle est transmise par le père. Ajoutons qu'elle se trouve dans une région du chromosome 17 dont la duplication uniparentale paternelle entraîne la mort des embryons qui la portent. Ces données avaient amené à faire l'hypothèse que la région délétée dans la mutation $\mathrm{T}^{\mathrm{h} p}$ contiendrait un gène essentiel dont l'expression serait assurée uniquement par l'allèle maternel. C'est ce raisonnement qui a guidé les expériences de Barlow et al. et leur a permis de démontrer que le gène de l'IGF2 - $r$ (qui code pour le récepteur de l'IGF2), et lui seul parmi quatre gènes repérés dans la région couverte par la délétion $\mathrm{T}^{\mathrm{h} p}$, 


\section{RÉFÉRENCES}

1. Crousc, HV. The controlling element in sex chromosome bchavior in Sciara. Genetics $1960 ; 45: 1429-43$

2. McGrath J, Solter D. Nuclear transplantation in the mousc embryo by microsurgery and ccll fusion. Science $1983 ; 220: 1300-2$.

3. McGrath J, Solter D. Complementation of mousc embryogencsis requires both the maternal and paternal genomes. Cell 1984 ; 37 : $179-83$.

4. Solter D. Differential imprinting and expression of maternal and paternal genomes. Annu Rev Genet, 1988 ; 22 : 127-46.

5. Babinct C, Barra J, Renard JP. Le marquage ct l'expression différenticls des génomes paternel ct materncl. médecine/sciences $1989 ; 5: 8-15$.

6. Paldi A, Jami J. L'empreinte génomique : complémentarité fonctionnclle de deux génomes parcntaux. médecine/sciences 1991 ; 7 : 247-54.

7. Cattanach BM, Becchcy CV. Autosomal and X-chromosomc imprinting. Development 1990 ; (suppl.) : 63-72.

8. Junien C, Henry I. Bras court du chromosome 11: empreinte parentalc différenticllc, tumorigenc̀se ct pertes d'allèles. médecine/sciences $1991 ; 5$ : 480-8.

9. Dreyfus JC. Quand deux chromosomes homologues viennent d'un scul parent... les disomics uniparentales en pathologic. médecine/sciences $1990 ; 6: 57-60$.

10. Surani MAH. Evidences and consequences of differences between maternal and paternal genomes during embryogencsis in the mousc. In : Rossant J, Pedersen RA, cds. Experimental Approaches to Mammalian Embryonic Development. Cambridgc : Cambridge University press, 1986 : 401-35.

11. Surani MAH, Barton SC, Howlett SK, Norris ML. Influence of chromosomal determinants on developpment of androgenetic and parthenogenetic cells. Development 1988 ; $103: 171-8$.

12. Mann JR, Gadi J, Harbison ML. Abbondanzo SJ, Stewart CL. Androgenctic mouse embryonic stem cells are pluripotent and cause skeletal defects in chimeras: implications for genctic imprinting. Cell $1990 ; 62$ : 251-60.

13. Barton SC, Ferguson-Smith AC, Fundelc R, Surani MA. Influcnce of paternally imprinted genes on development Development, $1991 ; 113$ : 679-88.

14. Fundelc RH, Norris ML, Barton SC, et al. Temporal and spatial sclection against parthenogenctic cells during development of fetal chimcras. Development 1990; 108 : 203-11.

$\mathrm{m} / \mathrm{s} n^{\circ} 1$, vol. 8, janvier 92

\section{* GLOSSAIRE}

15. Nagy A, Sass M, Markkula M. Systcmatic non-uniform distribution of parthenogenctic cells in adult mousc chimacras. Development $1989 ; 106$ : 321-4.

16. Surani MA, Reik W Allen D. Transgenes as molccular probes for genomic imprinting. Trends Genet $1988 ; 4$ : 59-62.

17. Relk W, Howlett SK Surani MA Imprinting by DNA methylation : from transgenes to endogenous gens sequences. Development 1990 ; (suppl.) : 99-106.

18. Sapienza C, Paquettc J. Tran TH, Peterson A. Epigenetic and genetic factors affect transgenc methylation imprinting. Development 1989 ; 107 : 165-68.

19. Engler P, Haasch D, Pinkert CA, et al. A strain-specific modificr on mousc chromosome controls the methylation of independent transgene loci. Cell $1991 ; 65$ : 939-47.

20. Surani MA, et al. Genome imprinting and development in the mousc. Development 1990 (suppl.) ; 89-98.

21. McGowan R, Campbcll R, Pcterson A, Sapienza C. Cellular mosaicism in the methylation and expression of hernizygous loci in the mouse. Genes 1989 ; 3 : 1669-76.

22. Chaillet JR, Vogt TF, Bcicr DR, Leder $P$. Parental-specific methylation of an imprinted transgenc is established during gametogenesis and progressively changes during embryogenesis. Cell 1991; 66 : 77-83.

23. DeChiara TM, Robcrtson EJ Efstratiadis A. Parental imprinting of the mousc insulin-like growth factor II gene. Cell 1991 ; $64: 849-59$

24. Barlow DP, Stöger R, Herrmann BG, Saito K, Schwelfer N. The mousc insulinlike growth factor typc-2 receptor is imprinted and closely linked to the Tme locus. Nature $1991 ; 349$ : 84-7.

25. Bartolomei MS, Zemel S, Tilghman SM. Parental imprinting of the mousc H19 genc. Nature 1991; 351 : 153-4.

26. Dreyfus JC. Gène H19 de la souris, cmpreinte parcntalc ct syndrome de Bcckwith-Wicdcmann. médecine/sciences 1991 ; 7, p. $746-7$

27. Babinet C, Kahn A. Empreinte parentalc : expression exclusive du gènc maternel du réccptcur d'IGF2. médecine/sciences $1991 ; 7: 292-4$.
Zygote : cellule fondatrice d'un organisme, née de la fécondation du gamète femelle, l'ovocyte par un gamète mâle, le spermatozoïde.

Pronoyau : désigne les noyaux qui se forment juste après la fécondation de l'ovocyte par un spermatozoïde, à partir du noyau de l'ovocyte et de la tête du spermatozoïde. A ce stade, qui est de courte durée (quelques heures chez la souris), les génomes parentaux sont physiquement séparés.

Chimère : individu né de la fusion de deux (ou plus) embryons de génotypes différents.

- Chimère par agrégation: les deux embryons sont agrégés pendant la période de clivage.

- Chimere par injection : une ou plusieurs cellules embryonnaires sont injectées dans la cavité d'un blastocyste.

Parthénogénotes : embryons provenant de l'activation spontanée ou artificielle d'ovocytes, sans participation d'un gamète mâle. Ils renferment donc un génome d'origine uniquement femelle.

Androgénotes : embryons obtenus par retrait, après la fécondation, du pronoyau femelle. Ces embryons possèdent donc uniquement un génome d'origine paternelle.

n'était exprimé que lorsqu'il était porté par le chromosome maternel. Fait intéressant, les trois gènes voisins sont exprimés également par l'allèle paternel et l'allèle maternel, ce qui montre que l'empreinte doit être très localisée [24, 27].

Dans le cas du gène $I G F 2$, ce sont des expériences de mutagénèse dirigée par recombinaison homologue dans les cellules souches embryonnaires qui ont permis de suspecter que ce gène était soumis à une empreinte génomique parentale. En effet, lorsque des mâles chimériques, constitués en partie de cellules portant une mutation nul dans l'IGF2 ont été croisés avec des femelles normales, dans tous les cas où l'allèle muté était transmis les souris présentaient un phénotype nain ; en outre, la quan- 
tité des ARN messagers de l'IGF 2 chez les hétérozygotes pour la mutation était bcaucoup plus faible que cellc attenduc, ce qui suggère que l'allèle matcrncl sauvage était particllement ou totalement inactif. Les études ultéricures, tant génétiques que moléculaires, ont permis de confirmer ces résultats et de démontrer sans ambiguïté que, pour ce gène scul l'allèle paternel est exprimé tandis que l'allèle matcrncl est silencieux. Ajoutons que lc gène $I G F 2$, localisé sur le chromosome 7 de la souris est très proche du gènc $H 19$; or, ccs deux gènes ont une empreinte dc typc opposé (l'allèle maternel étant actif pour $H 19$, l'allèle paterncl pour $I G F$ 2), ce qui vient renforcer l'idéc que les mécanismes qui gouvernent l'cmpreinte agissent de manière très localiséc.

Il cst clair que la mise en évidence de gènes soumis à l'cmprcinte génomique parentalc constitue un pas décisif; non seulement ces études confirment la validité des interprétations faitcs à partir de l'cxamen du développement des cmbryons uniparentaux, mais elles ouvrent la voic à l'étude des mécanismes moléculaires de ccttc emprcinte.

\section{Charles Babinet}

Unité de génétique des mammiferes, Institut Pasteur, 25, rue du DocteurRoux, 75724 Paris Cedex 15, France.

\section{Chroniques génomiques

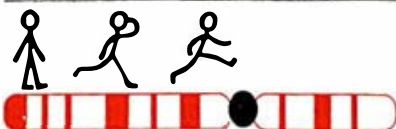 \\ HUGO 1992 (suite...) \\ par Bertrand JORDAN}

Pas de nouvelle Chronique Génomique ce mois-ci : l'auteur, de nouveau basé à Marseille et soumis à de multiples sollicitations, n'a pas réussi à trouver la disponibilité requise. Ce n'est que partie remise, et les chroniques continueront dès le mois prochain!

Les quelques lignes qui suivent sont destinées à rectifier une information qui, ajoutée en dernière minute à la Chronique précédente, est devenue inexacte. II s'agit de la désignation de Norton Zinder comme secrétaire général à plein temps de l'organisation, qui paraissait alors acquise. En fait la décision finale de HUGO a été de ne pas procéder à cette nomination, en considérant que les ressources disponibles ne permettaient pas d'employer une telle personnalité de façon efficace et à plein temps. Comme l'existence de ces négociations et l'identité de l'éventuel secrétaire général avaient fait l'objet de nombreux commentaires, HUGO tient à préciser que cette décision est uniquement motivée par des considérations logistiques et financières et ne met nullement en cause les qualités et la compétence de Norton Zinder. A titre personnel, j'ajouterai que ce petit incident de parcours montre bien les difficultés éprouvées pour " asseoir " une organisation dont les contours sont encore un peu nébuleux, le financement limité, et qui a pourtant besoin de s'assurer des concours multiples pour jouer son rôle. Le devenir de HUGO, est-il besoin de le rappeler, c'est aussi notre affaire, et notre responsabilité...

\section{TIRÉS A PART}

